Relations industrielles

Industrial Relations

\title{
L'univers politique et syndical des cadres, par Gérard Grunberg et René Mouriaux, Paris, Presses de la Fondation Nationale des Sciences Politiques, 1979, 230 pp.
}

\section{Christian Buisson}

Volume 35, numéro 2, 1980

URI : https://id.erudit.org/iderudit/029075ar

DOI : https://doi.org/10.7202/029075ar

Aller au sommaire du numéro

Éditeur(s)

Département des relations industrielles de l'Université Laval

ISSN

0034-379X (imprimé)

1703-8138 (numérique)

Découvrir la revue

Citer ce compte rendu

Buisson, C. (1980). Compte rendu de [L'univers politique et syndical des cadres, par Gérard Grunberg et René Mouriaux, Paris, Presses de la Fondation Nationale des Sciences Politiques, 1979, 230 pp.] Relations industrielles / Industrial Relations, 35(2), 346-348. https://doi.org/10.7202/029075ar

Tous droits réservés (C) Département des relations industrielles de l'Université Laval, 1980
Ce document est protégé par la loi sur le droit d'auteur. L’utilisation des services d'Érudit (y compris la reproduction) est assujettie à sa politique d'utilisation que vous pouvez consulter en ligne.

https://apropos.erudit.org/fr/usagers/politique-dutilisation/ 
Il faut féliciter l'auteur d'avoir produit ce dictionnaire qui est un instrument indispensable aux hommes d'affaires, étudiants, journalistes, fonctionnaires et même au public en général et qui mérite la plus large diffusion possible.

\section{Gérard DION}

Université Laval

L'univers politique et syndiçal des cadres, par Gérard Grunberg et René Mouriaux, Paris, Presses de la Fondation Nationale des Sciences Politiques, 1979, 230 pp.

L'instrument de cette recherche est un sondage effectué en mai/juin 1974 auprès d'un échantillon de 1481 cadres de l'industrie et des services. Définie à partir du classement INSEE (ingénieurs et cadres administratifs supérieurs, techniciens et cadres administratifs moyens), la population étudiée s'organise en fonction de six variables: sexe, âge, qualification, taille des établissements, branches, régions.

En tenant compte du contexte de l'époque, de l'incidence de l'actualité sur les réponses - la datation, évitée dans la majorité des questions, est parfois utilisée comme repère - les auteurs ont voulu dégager les lignes de force qui sous-tendent les comportements des cadres. Leur propos n'était pas de fixer l'image définitive et complète de l'opinion des cadres au lendemain de l'élection de V. Giscard d'Estaing, mais d'en éclairer la cohésion et la dispersion, les déterminants et les tendances. C'est cette orientation de recherche, interrogeant les données de l'observation et les situant dans leur durée, qui valide les conclusions et les hypothèses présentées dans ce livre, à partir de matériaux rassemblés en 1974.

Avant d'aborder l'analyse des critères d'identité des cadres, les auteurs soulignent une première difficulté: la diversité des problématiques qui tentent de les situer. Procédant par larges regroupements, ils relèvent trois types d'approche, dont chacun est d'ailleurs loin d'être univoque.
La première fait appel à la notion de classe moyenne ou intermédiaire. D'Aristote à V. Giscard d'Estaing, une rétrospective rapide emprunterait à Guizot, Gambetta, Jaurès, la C.G.C., J. Royer, J. Chirac. Traditionnellement gratifiée de qualités de compétence et de mesure, la classe moyenne est vouée à un rôle médiateur. En ce sens, son influence ne peut que s'accroître, d'autant que l'évolution des conditions de vie entraîne, selon l'auteur de Démocratie française, «l'expansion d'un immense groupe central aux contours peu tranchés".

Les auteurs marxistes (C. Baudelot, R. Establet, N. Poulantzas) placent les cadres dans le champ d'affrontement des classes. Groupe fissuré par la bi-polarisation, traversé de courants contradictoires, d'idéologies qui le pénètrent de l'extérieur sans qu'il puisse, pour son propre compte, en sécréter aucune. Les cadres, dépourvus d'identité idéologique, n'ont pas d'avenir de classe.

Une troisième approche, selon A. Touraine, R. Dahrendorf, J.K. Galbraith, D. Bell, notamment, aussi différenciée que les précédentes, récuse la bi-polarisation marxiste en raison de l'évolution technique modifiant en profondeur les rapports de production et la structure sociale. La société postindustrielle multiplie les pouvoirs partiels et les intérêts spécifiques. Société de compromis et de turbulences, la compétence technique s'y affirme comme source de prééminence, rivale des légitimités instituées par la propriété et le pouvoir politique.

Chacune des thèses prend appui sur une délimination plus ou moins précise du groupe des cadres; toutes mettent en cause le classement INSEE en proposant d'autres coupes de population. G. Grunberg et R. Mouriaux ont cependant suivi l'INSEE pour explorer la relation entre les catégories 3 et 4 dont le rapprochement ne leur paraît pas neutre, mais aussi en raison du vaste terrain ainsi ouvert à l'influence des facteurs de cohésion et de fragmentation. Y a-t-il une conscience commune de spécificité d'intérêts reliant tous ceux qui appartiennent à cet ensemble? Les clivages qui le segmentent s'expriment-ils en 
idéologies et comportements distincts? Quelles stratégies s'en dégagent, en admettant qu'il s'en constitue d'autonomes: médiation, alliances privilégiées, assimilation?

Pour appréhender la structuration du groupe et son positionnement social, six indicateurs sont proposés:

Le premier est la position dans la hiérarchie des rémunérations. Celle-ci conditionne le sentiment plus ou moins affirmé d'une proximité soit avec les dirigeants, soit avec les exécutants. Aux niveaux extrêmes de l'échantillon, elle se traduit par des choix politiques et syndicaux sans ambiguïté. Aux rémunérations moyennes correspondent par contre des options moins affirmées et des attitudes de repliement: corporatisme, attachement au statut de cadre, apolitisme.

D'autres données interviennent également, qui permettent de nuancer l'analyse:

D'abord la place de la fonction dans l'organisation de l'entreprise. La position stratégique du cadre, son degré de sécurité ou de dépendance, l'incidence des changements techniques ou structurels sur la qualification de sa fonction, introduisent de multiples variables. L'approche des phénomènes est d'autant plus complexe qu'un même déterminant peut donner lieu à des comportements divers: l'insécurité, la mise en cause d'avantages acquis peuvent développer des mécanismes de défense catégorielle, mais aussi des recherches de stratégies indıviduelles ou des tendances solidaristes vis-à-vis des autres salariés.

La formation (écoles, université, diplômes de techniciens) outre le fait qu'elle définit des appartenances distinctes, détermine également des formes et degrés de dépendance à l'égard de l'entreprise et des comportements sociaux sensiblement différents.

L'exercice du commandement favorise le développement du loyalisme voire l'identification avec les dirigeants. À l'inverse, dans les secteurs à forte concentration de cadres spécialisés, écartés de la prise de décision comme de la relation d'autorité, on constate une tendance à se rapprocher des exécutants.
Enfin interviennent les traditions familiales. La comparaison entre l'origine sociale et les attitudes syndicales et politiques fait apparaître une continuité dans la majorité des cas. Les choix politiques exprimés marquent des évolutions (glissement du centre droit vers les frontières socialistes) mais pratiquement pas de rupture.

Selon G. Grunberg et R. Mouriaux, la multiplicité des variables aboutit en définitive à quatre types de comportements: aux extrêmes se situent les légitimistes et les solidaristes.

Implantés surtout dans les entreprises moyennes ou les industries à faible taux d'encadrement, les légitimistes bénéficient généralement de relations directes et fréquentes avec les dirigeants. Résolument hostiles à toute forme de syndicalisation des cadres, leur adhésion à l'entreprise repose sur l'assimilation sans réserve au patronat. Les solidaristes sont des cadres jeunes, d'origine sociale modeste, occupant des fonctions de niveau moyen dans des entreprises où le syndicalisme existe. Leurs modèles de comportement ne se différencient pas de ceux des salariés.

Entre eux se trouve une population aux caractères moins nettement contrastés, dont les tendances s'orientent vers deux modèles: les alliés, les individualistes. Les alliés, favorables au syndicalisme catégoriel sont des hommes de compromis qui négocient leur appui. Les individualistes récusent la syndicalisation et restent aussi distants à l'égard du patronat que des salariés.

Ainsi se distribuent les comportements des cadres. Groupe éclaté? à tout le moins hétérogène, comme le constatent les auteurs. Il est vrai qu'ils ont assigné des frontières particulièrement larges à la population étudiée. On peut regretter le choix du classement INSEE dont les définitions des cadres moyens et supérieurs ne recouvre qu'imparfaitement celles de la plupart des entreprises, des conventions collectives et des caisses de retraite de cadres. Le problème des définitions mériterait une étude particulière en raison de ce qu'il recouvre. Certes, les frontières sont parfois floues, mais il est d'autant plus néces- 
saire d'éviter tout risque de confusion qui ajouterait encore à la complexité des phénomènes observés. L'un des mérites de cette recherche est d'avoir pris en compte cette complexité en une démarche extensive qui élargit à mesure ses hypothèses et s'enrichit de nouvelles problématiques.

Le constat d'hétérogénéité auquel elle aboutit n'est pas fait pour surprendre. La question qui se profile est celle des deux dimensions selon lesquelles un groupe vit son identité socio-professionnelle. C'est par les transactions entre les déterminants sécrétés par l'entreprise et ceux que fait peser l'environnement que l'on pourrait approfondir l'interrogation. Cela reviendrait à étudier non plus seulement un groupe, sachant que les phénomènes que l'on saisit ainsi ont un caractère quelque peu abstrait, mais aussi des situations constituant des réalités sociales limitées mais complètes.

En d'autres termes, c'est en confrontant de multiples coupes effectuées sur le terrain que l'on pourra compléter les problématiques et éprouver la validité des hypothèses. Les cadres, enjeu politique? Il est significatif que ce soit là encore une question qui se pose nécessairement de l'extérieur. On pourrait la reformuler dans un sens qui la resitue: les cadres, objets ou acteurs du politique?

\section{Christian BUISSON}

Institut d'administration des entreprises Paris

Le mouvement de restructuration du travail, par Paul Dechêne, Québec, Ministère du Travail et de la Main-d'oeuvre, coll. études et recherches, $187 \mathrm{pp}$.

Cette publication du ministère du Travail et de la Main-d'oeuvre constitue un outil pédagogique des plus intéressants pour des personnes désireuses de se familiariser avec les problèmes liés à l'organisation du travail et à son évolution. L'ouvrage est divisé en deux parties: une analyse thématique de la littérature existante et une bibliographie sélective remarquablement commentée.
L'auteur établit tout d'abord son propre bilan de la littérature consacrée à la réorganisation du travail. Constatant que l'ampleur de cette littérature témoigne de la vigueur du débat soulevé par les nouvelles formes d'organisation du travail, il ne se limite pas à en établir un "bilan-portrait». Son intention est de formuler une synthèse critique de cette littérature «tant de cerner les pièges et les limites des réformes apportées à l'organisation du travail, que d'évaluer les conséquences de ces expériences pour les travailleurs, pour l'action syndicale». Il y réussit assez bien quoiqu'il ne lui soit pas possible, en si peu de pages, d'approfondir les problèmes qu'il soulève à juste titre. Le lecteur demeure donc sur sa faim.

Dans la première partie de l'ouvrage, l'auteur insiste sur la crise actuelle de l'organisation du travail, particulièrement sur les signes d'une résistance accrue des travailleurs à l'OST. Il décrit les approches les plus populaires en matière de restauration du travail enrichissement des tâches, approche sociotechnique et groupes autonomes - et s'attarde sur l'ampleur et l'impact de ces expériences. Il tente d'en évaluer les résultats pour l'entreprise et pour le travailleur et s'intéresse aux conséquences de ces tentatives sur l'action syndicale. De façon générale, l'analyse de Dechêne est critique mais nuancée. À l'exemple d'Annie Borzeix, il désire "échapper au double piège d'un réformisme à la fois naîf et optimiste, et d'un idéalisme révolutionnaire qui se contente de renvoyer à plus tard la résolution du problème soulevé». Cette attitude de prudence est de mise dans une revue de littérature, surtout si elle est appelée à jouer un rôle d'initiation au sujet dans divers milieux.

On peut difficilement reprocher à ce genre de publication de demeurer trop général. Le format et l'intention l'y obligent. Par contre, le lecteur désireux d'approfondir quelques uns des thèmes mentionnés précédemment, trouvera dans la seconde partie de l'ouvrage une bibliographie sélective soigneusement commentée par l'auteur. De Taylor et Friedmann à Trist et Davis, en passant par les théories sur la satisfaction au travail et leur 